\title{
Enterovirus 71 VP1 promotes mouse Schwann cell autophagy via ER stress-mediated PMP22 upregulation
}

\author{
PEIQING LI ${ }^{1}$, SIDA YANG ${ }^{2}$, DANDAN HU ${ }^{2}$, DAN WEI $^{3}$, JING LU $^{4}$, HUANYING ZHENG $^{4}$, \\ SHUSHAN NIE ${ }^{1}$, GUANGMING LIU ${ }^{1}$ and HAOMEI YANG ${ }^{1}$
}

\begin{abstract}
${ }^{1}$ Department of Pediatric Emergency; ${ }^{2}$ Department of Pediatric Neurology, Guangzhou Women and Children's Medical Center, Guangzhou Medical University, Guangzhou, Guangdong 510623; ${ }^{3}$ Paediatric Intensive Care Unit, First Affiliated Hospital of Guangxi Medical University, Nanning, Guangxi 530021; ${ }^{4}$ Guangdong Provincial Institution of Public Health, Guangdong Provincial Center for Disease Control and Prevention, Guangzhou, Guangdong 511430, P.R. China
\end{abstract}

Received September 25, 2018; Accepted May 28, 2019

DOI: $10.3892 /$ ijmm.2019.4218

\begin{abstract}
Enterovirus 71 (EV71) accounts for the majority of hand, foot and mouth disease-related deaths due to fatal neurological complications. EV71 structural viral protein 1 (VP1) promotes viral replication by inducing autophagy in neuron cells, but the effect of VP1 on myelin cells is unclear. The present study aimed to investigate the role and mechanism of VP1 in autophagy of mouse Schwann cells. An EV71 VP1-expressing vector (pEGFP-C3-VP1) was generated and transfected into mouse Schwann cells. Transmission electron microscopy and western blot analysis for microtubule-associated protein 1 light chain $3 \alpha$ (LC3) II (an autophagy marker) were used to assess autophagy. Reverse transcription-quantitative PCR and immunofluorescence were performed to determine the expression of peripheral myelin protein 22 (PMP22). Small interfering RNA against PMP22 was used to investigate the role of PMP22 in mouse Schwann cell autophagy. Salubrinal [a selective endoplasmic reticulum (ER) stress inhibitor] was used to determine whether PMP22 expression was affected by ER stress. The present results indicated that VP1 promoted mouse Schwann cell autophagy. Overexpression of VP1 upregulated PMP22. PMP22 deficiency downregulated LC3II and thus inhibited autophagy. Furthermore, PMP22 expression was significantly suppressed
\end{abstract}

Correspondence to: Dr Sida Yang, Department of Pediatric Neurology, Guangzhou Women and Children's Medical Center, Guangzhou Medical University, 9 Jinsui Road, Guangzhou, Guangdong 510623, P.R. China

E-mail: yangsida2013@126.com

Abbreviations: CNS, central nervous system; ER, endoplasmic reticulum; EV71, enterovirus 71; HFMD, hand, foot and mouth disease; PMP22, peripheral myelin protein 22; SAL, salubrinal; siRNA, small interfering RNA; TEM, transmission electron microscopy; VP1, viral protein 1

Key words: enterovirus 71 structural viral protein 1, autophagy, endoplasmic reticulum stress, peripheral myelin protein 22 by salubrinal. In conclusion, VP1 promoted mouse Schwann cell autophagy through upregulation of ER stress-mediated PMP22 expression. Therefore, the VP1/ER stress/PMP22 autophagy axis may be a potential therapeutic target for EV71 infection-induced fatal neuronal damage.

\section{Introduction}

Enterovirus 71, a single-stranded RNA virus, is one of the major causative pathogens of contagious hand, foot and mouth disease (HFMD) that mainly affects children under the age of $5(1,2)$. HFMD is an emerging public health issue worldwide, especially in Asia-Pacific countries $(1,3,4)$. Although HFMD is considered as a self-limited disease characterized by ulcerating vesicles in the mouth and viral rashes on hands and feet (5-7), a small proportion of cases are severe and even fatal due to cardiopulmonary or neurological complications $(8,9)$. EV71 infection accounts for $\geq 80 \%$ of severe cases of HFMD and $90 \%$ of HFMD-related deaths in China (10). Increasing evidence indicates that EV71 may target neurons in the central nervous system (CNS), leading to neuronal degeneration, severe neurological disorders and even death (11-13). A previous study by our group demonstrated that neuronal necrosis and neuronophagia were present in the brainstem in fatal EV71-infected cases (14). Despite the neurotropic characteristics of EV71, the pathogenesis and molecular mechanisms of EV71-induced neuronal damage remain largely unknown. EV71 possesses four structural proteins including structural viral protein (VP) 1, VP2, VP3 and VP4. VP1 homodimers are the main component of the characteristic icosahedral capsid contributing to the pathogenicity and stability of the EV71 virus to survive in the environment of the gastrointestinal tract $(15,16)$.

Autophagy is a cellular process mediated by a unique organelle named autophagosome that transports cytoplasmic components to the lysosomes for degradation $(17,18)$. Changes in autophagy in the nervous system is associated with various neurodegenerative and neurometabolic disorders, such as Alzheimer's disease and Niemann-Pick disease (19-21). Autophagy can be observed by transmission electron microscopy (TEM) and assessed by measuring the conversion of the microtubule-associated protein 1 light chain $3 \alpha$ (LC3) to 
phosphatidylethanolamine-conjugated LC3 (LC3II) localized in autophagosome membranes, which reflects the number of autophagosomes or the degree of autophagy (22-24). EV71 has been demonstrated to induce autophagy in infected human rhabdomyosarcoma and neuroblastoma cells $(25,26)$. A previous study by our group demonstrated that EV71 VP1 also induces autophagy in cultured primary EV71-infected brainstem neurons, which can be inhibited by salubrinal (SAL), an inhibitor of endoplasmic reticulum (ER) stress (27), suggesting an essential role of ER stress in VP1-induced autophagy.

ER stress is triggered by the accumulation of unfolded or misfolded proteins in the ER $(28,29)$. Although the relationship between ER stress and autophagy is not yet fully understood, it is well established that there is a dynamic crosstalk between these two systems, and ER stress either stimulates or inhibits autophagy $(28,30,31)$. Since ER stress and autophagy are commonly concurrent in some human pathologies, such as cardiovascular diseases, cancers and neurodegenerative disorders (31-33), it is of great importance to identify ER stress-associated factors as positive or negative regulators of autophagy. Peripheral myelin protein 22 (PMP22) is a transmembrane glycoprotein highly expressed in the myelinating Schwann cells of peripheral neurons, and contributes to synthesis and function of myelin sheaths (34). In Schwann cells, newly synthesized PMP22 is transiently retained in ER and Golgi before being transported to the plasma membrane $(35,36)$. Under pathological conditions, excessive mature or premature (unfolded or misfolded) PMP22 accumulates in the ER and interacts with calnexin, a $\mathrm{Ca}^{2+}$-binding chaperone, leading to ER retention and activation of ER stress $(37,38)$. Nevertheless, it remains unknown whether the relationship between PMP22 and ER stress is associated with autophagy.

The present study hypothesized that PMP22 may be a downstream effector of ER stress and may trigger the activation of autophagy in response to the EV71 capsid protein VP1. To examine this hypothesis, mouse Schwann cells were transfected with VP1-expressing vectors, and the effect of VP1 overexpression in autophagy and PMP22 expression was explored. The results suggested that ER stress induced the expression of PMP22 and that it was essential for autophagy of mouse Schwann cells, suggesting an involvement of the VP1/ER stress/PMP22 axis in the autophagy regulation of mouse Schwann cells. Targeting the VP1/ER stress/PMP22 axis may serve in the future as a novel therapeutic strategy against EV71 infection-induced neuronal damage.

\section{Materials and methods}

Cell line and culture. Mouse Schwann cells were purchased from ScienCell Research Laboratories, Inc. and maintained in Schwann cell medium (ScienCell Research Laboratories, Inc.) containing penicillin $(100 \mathrm{U} / \mathrm{ml})$ and streptomycin $(100 \mu \mathrm{g} / \mathrm{ml}$; Hyclone; GE Healthcare Life Sciences) in poly-L-lysine-coated $\left(2 \mu \mathrm{g} / \mathrm{cm}^{2}\right)$ flasks at $37^{\circ} \mathrm{C}$ in a humidified atmosphere of $5 \% \mathrm{CO}_{2}$.

EV71 was isolated from clinical specimens including throat, anal swabs and stools of a patient with HFMD due to EV71 infection, provided by the Center for Disease Control and Prevention of Guangdong Province (Guangzhou, China). The patient was diagnosed by Guangxi Medical University
(Nanning, China) based on pathological analysis by the Forensic Identification Center,Zhongshan School of Medicine, Sun Yat-sen University (Guangzhou, China).

The study was approved by the Ethics Committee of the Center for Disease Control and Prevention of Guangdong Province (no. 2017020812). All procedures performed involving human participants were in accordance with the ethical standards of the institutional and/or national research committee and with the 1964 Helsinki declaration and its later amendments or comparable ethical standards. Written informed consent was obtained from the family of the patient from whom EV71 was isolated for the use of their clinical samples for research purposes.

Gene cloning and transfection. Total RNA was extracted from EV71 using TRIzol (Thermo Fisher Scientific, Inc.) and reverse transcribed to obtain cDNA (cat. no. A3800; Promega Corporation). The 894-bp VP1 cDNA was synthesized by reverse transcription-polymerase chain reaction (RT-PCR; cat. no.2011A; Takara Bio, Inc.) using the primers: Forward 5'-CCGCTCGAGGCCACCATGGGTGATGGA ATTGCAGACATGA-3' and reverse 5'-CGCGGATCCTAG TGT TGTTATTTTGTCCCTACTTGTGC-3' (Genewiz, Inc.). The thermocycling conditions were: $95^{\circ} \mathrm{C}$ for $2 \mathrm{~min}$, followed by 30 cycles at $95^{\circ} \mathrm{C}$ for $10 \mathrm{sec}$ and $58^{\circ} \mathrm{C}$ for $30 \mathrm{sec}$, then $60^{\circ} \mathrm{C}$ for $5 \mathrm{~min}$. The PCR products were subcloned into the pEGFP-C3 expression vector (Clontech Laboratories, Inc.) and sequencing was performed by Sangon Biotech for confirmation of successful cloning. The results were compared with the VP1 cDNA sequence reported in the GenBank database (GenBank accession number: U55763). Cells were transiently transfected with $2 \mathrm{mg}$ plasmids using Lipofectamine 2000 (Invitrogen; Thermo Fisher Scientific, Inc.) for $24 \mathrm{~h}$, according to the manufacturer's instructions.

Small interfering RNA (siRNA). The siRNA against PMP22 (siPMP22; $100 \mathrm{nmol}$ ) was from Santa Cruz Biotechnology, Inc. (sc-42037) and transfected using the siRNA transfection reagent (Santa Cruz Biotechnology, Inc.; sc-37007). Scramble negative control siRNA (NCsiRNA; $100 \mathrm{nmol}$ ) was used as a control. The cells were transfected for $24 \mathrm{~h}$ prior to experiments.

Reverse transcription-quantitative $P C R(R T-q P C R)$. Total RNA was extracted from cells using TRIzol (Thermo Fisher Scientific, Inc.), according to the manufacturer's instructions, and was reverse transcribed into cDNA using reverse transcriptase (cat. no. A3800; Promega Corporation). qPCR was performed using SYBR Green qPCR SuperMix (cat. no. 11733046; Thermo Fisher Scientific, Inc.), with the following conditions: $95^{\circ} \mathrm{C}$ for $2 \mathrm{~min} ; 40$ cycles at $95^{\circ} \mathrm{C}$ for $10 \mathrm{sec}$ and $55^{\circ} \mathrm{C}$ for $30 \mathrm{sec}$; and final step $55^{\circ} \mathrm{C}$ for $10 \mathrm{~min}$. Primers sequences are listed in Table I. GAPDH was used as an internal control. Relative fold changes in mRNA expression were calculated using the formula $2^{-\Delta \Delta \mathrm{Cq}}(39)$.

Western blot analysis. Mouse Schwann cells were lysed using a RIPA lysis and extraction buffer (cat. no. 89900; Thermo Fisher Scientific, Inc). Protein concentration in the lysates was determined using the bicinchoninic acid protein assay reagent (Beyotime Institute of Biotechnology). Then, $50 \mathrm{ng}$ of 
Table I. Primers used for quantitative PCR.

\begin{tabular}{lll}
\hline Gene & Forward primer $\left(5^{\prime}-3^{\prime}\right)$ & Reverse primer $\left(5^{\prime}-3 '\right)$ \\
\hline PMP22 & CTGCCAGCTCTTCACTCTCA & GTTGACATGCCACTCACTGT \\
GAPDH & GGCCTCCAAGGAGTAAGAAA & GCCCCTCCTGTTATTATGG
\end{tabular}

PMP22, peripheral myelin protein 22.

proteins were separated by $10 \%$ SDS-PAGE and transferred to polyvinylidene fluoride membranes. The membranes were blocked with $5 \%$ nonfat milk powder in Tris-buffered saline/Tween 20 (TBST) at room temperature and incubated with anti-GAPDH: (1:1,000; Abcam; ca. no. ab181602) or anti-LC3II (1:1,000; Abcam; cat. no. ab51520) for 1-2 h at room temperature. Following three washes with cold TBST, the membranes were incubated with peroxidase-conjugated secondary antibody (1:4,000; Thermo Fisher Scientific, Inc.; cat. no. 31460) for $1 \mathrm{~h}$ at room temperature. After three washes with TBST, the protein expression was detected using an enhanced chemiluminescent development reagent (GE Healthcare) and densitometry analysis was performed using Quantity One (Bio-Rad Laboratories, Inc.).

Immunofluorescence. Mouse Schwann cells were seeded on sterile coverslips $48 \mathrm{~h}$ after transfection and incubated overnight at $37^{\circ} \mathrm{C}$. Cells were fixed with $4 \%$ paraformaldehyde for $30 \mathrm{~min}$, followed by incubation with $0.2 \%$ Triton $\mathrm{X}-100$ at $4^{\circ} \mathrm{C}$ for $5 \mathrm{~min}$. After PBS washes, the cells were blocked with $10 \%$ normal goat serum (Jackson ImmunoResearch Laboratories, Inc.) for $30 \mathrm{~min}$ and incubated with anti-PMP22 antibody (1:200; Abcam; cat. no. ab211052) overnight at $4^{\circ} \mathrm{C}$. The cells were incubated with fluorescence-conjugated secondary antibodies $(1: 1,000$; Thermo Fisher Scientific, Inc.; cat. no. 35510) for $1 \mathrm{~h}$ at room temperature. In addition, Fluo-8 AM (1:100; cat. no. 21080; AAT Bioquest, Inc.), a green fluorescent indicator that monitors $\mathrm{Ca}^{2+}$ conncetration and flux in cells, was used as a positive control for ER stress inhibition by SAL. The images were captured with a Leica confocal microscope (Leica Microsystems GmbH).

Transmission electron microscopy (TEM) analysis. Mouse Schwann cells were prefixed with $2.5 \%$ glutaraldehyde for $2 \mathrm{~h}$ and post-fixed with $1 \%$ osmic acid for $2 \mathrm{~h}$ at $4{ }^{\circ} \mathrm{C}$, followed by gradient dehydration in 30, 50 and $70 \%$ ethanol (10 min each), then 80,90 and $95 \%$ acetone (10 min each), and $100 \%$ acetone (10 min twice). The cells were embedded in resin at $35^{\circ} \mathrm{C}$, $45^{\circ} \mathrm{C}, 60^{\circ} \mathrm{C}$ for $24 \mathrm{~h}$ respectively, then sectioned to $60 \mathrm{~nm}$ thick, and stained with lead citrate for $15 \mathrm{~min}$ at room temperature. The stained cells were observed and imaged under a Hitachi H-7500 transmission electron microscope (Hitachi, Ltd.).

Statistical analysis. All experiments were repeated at least three times. Data are expressed as mean \pm standard error (SE). Statistical significance was assessed by the Student's $t$ test or one-way ANOVA with the Least Significance Difference post hoc test, using the SPSS 16.0 statistical software (SPSS, Inc.). $\mathrm{P}<0.05$ was considered to indicate a statistically significant difference.

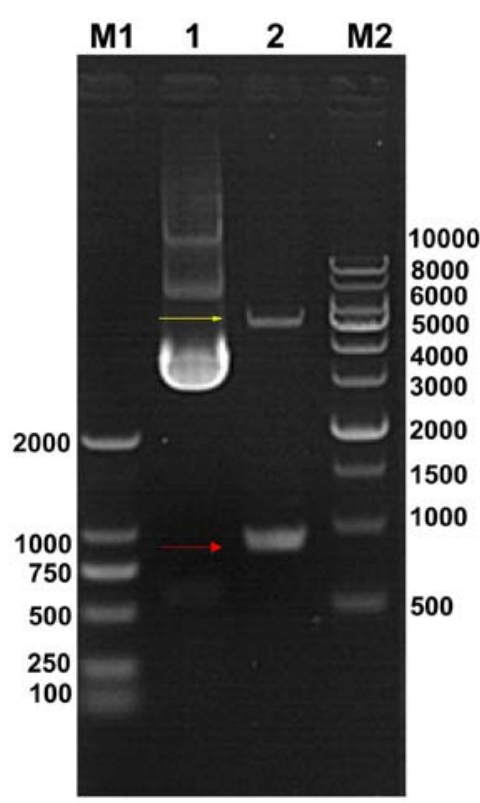

Figure 1. Cloning and confirmation of VP1 cDNA. Image from agarose electrophoresis analysis of intact (lane 1) and restriction enzyme-digested (lane 2) pEGFP-C3 plasmids with cloned VP1 cDNA. The red arrow points to the VP1 cDNA band, while the yellow arrow points to the linear empty vector band. VP1, structural viral protein 1; M, DNA marker.

\section{Results}

Cloning and identification of VPI cDNA. To determine if the VP1 cDNA was successfully cloned into the pEGFP-C3 vector, plasmids from transformed bacteria were prepared and digested with $B a m \mathrm{HI}$ and XhoI. The results of agarose electrophoresis demonstrated that a band was located between 750 and 1,000 bp (Fig. 1), which is consistent with the size of the VP1 cDNA (894 bp), according to the GenBank database. The sequencing results also indicated that the cloned fragment was identical to the VP1 cDNA sequence (data not shown), suggesting that VP1 cDNA was successfully cloned into the vector, without any mutation.

Overexpression of VPl activates mouse Schwann cell autophagy. To examine whether VP1 has an effect on mouse Schwann cell autophagy, the cellular and subcellular morphology of VP1-overexpressing mouse Schwann cells was analyzed using TEM. As presented in Fig. 2, VP1-overexpressing mouse Schwann cells exhibited characteristic features of autophagy, including swelling mitochondria, dilation and degranulation of rough ER and vesicle-like dilation of Golgi (40). By contrast, the organelles in untransfected and empty vector-transfected control mouse Schwann cells 

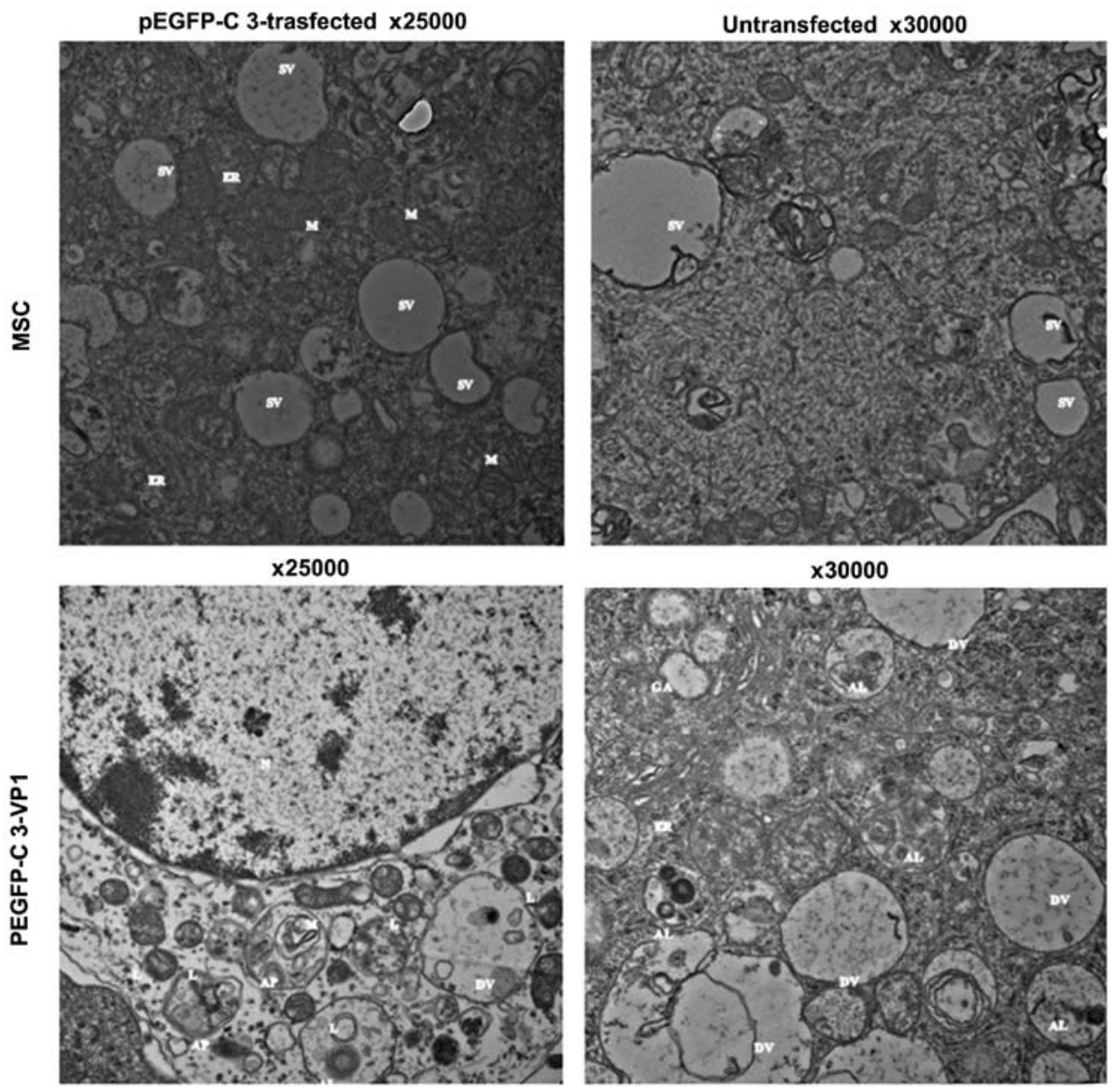

Figure 2. Effect of VP1 overexpression on mouse Schwann cell autophagy. Mouse Schwann cells were transfected with pEGFP-C3-VP1 for 48 h. Untransfected and pEGFP-C3-trasfected cells were used as blank and negative controls, respectively. Representative transmission electron microscopic images depicting subcellular structures of mouse Schwann cells are shown. VP1-overexpressing mouse Schwann cells exhibited the features of autophagy such as swelling mitochondria, dilation and degranulation of rough ER, and vesicle-like dilation of Golgi, whereas the organelles in untransfected and pEGFP-C3-transfected control mouse Schwann cells were still morphologically normal. VP1, structural viral protein 1; N, nucleus; M, mitochondrion; L, lysosome; AP, autophagosome; AL, autolysosome; DV, degradation vesicles; GA, Golgi apparatus; ER, endoplasmic reticulum; SV, secretory vesicles; MSC, mouse Schwann cell.

were morphologically normal (Fig. 2). These results suggested that VP1 may activate autophagy in mouse Schwann cells.

Overexpression of VP1 upregulates PMP22 expression in mouse Schwann cells. A previous study from our group has indicated an essential role of ER stress in VP1-induced autophagy in primary cultured EV71-infected brainstem neurons (14). Because PMP22 is abundant in Schwann cells and associated with ER stress activation $(34,37,38)$, the present study hypothesized that PMP22 might correlate with VP1 and serve an important role in VP1-induced autophagy. To examine this hypothesis, the mRNA and protein expression levels of PMP22 were detected in VP1-overexpressing mouse Schwann cells. As presented in Fig. 3A, the mRNA expression levels of PMP22 were significantly increased in VP1-overexpressing mouse Schwann cells compared with empty vector-transfected cells. Immunofluorescence analysis demonstrated similar results (Fig. 3B). Finally, western blot analysis confirmed that PMP22 protein expression levels were significantly increased in VP1-overexpressing mouse Schwann cells compared with empty vector-transfected cells (Fig. 3C and D). These data indicated that VP1 was an upstream regulator of PMP22, suggesting a possible involvement of PMP22 in VP1-mediated activation of mouse Schwann cell autophagy.
PMP22 is essential for mouse Schwann cells autophagy. Next, the present study sought to investigate whether PMP22 may be involved in mouse Schwann cell autophagy. PMP22 was silenced by siRNA, as confirmed by significantly decreased expression of PMP22 in siPMP22-transfected mouse Schwann cells (Fig. 4A and B). Notably, compared with NCsiRNA-transfected groups, PMP22 silencing significantly downregulated the expression of LC3 isoform LC3II, an established autophagy marker (41), while VP1 overexpression reversed this phenomenon (Fig. 4A and B). Consistently, the ratio of LC3II to LC3I in PMP22-deficient mouse Schwann cells was also significantly lower compared with the NCsiRNA-transfected groups (Fig. 4C). Compared with the negative control group, SAL treatment alone decreased PMP22 and increased LC3II expression, while siPMP22-transfected cells exhibited decreased PMP22 and LC3II expression (Fig. 4). Furthermore, TEM images revealed that there was no observable autophagic structure in siPMP22-transfected mouse Schwann cells compared with NCsiRNA-transfected cells (Fig. 5). Taken together, these data suggested that PMP22 was required for the VP1-mediated activation of autophagy in mouse Schwann cells.

ER stress induces PMP22 expression in mouse Schwann cells. Since PMP22 is closely associated with ER stress and 
A

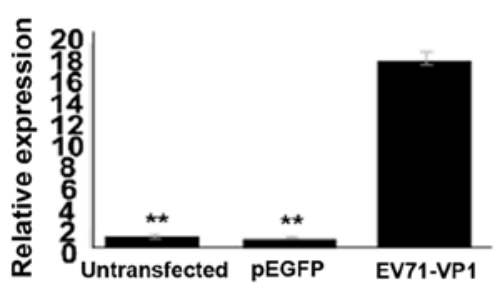

C

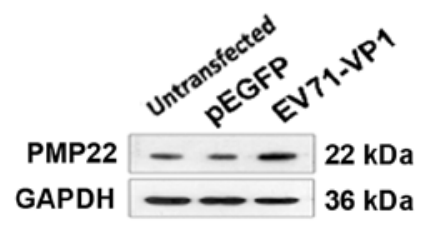

D

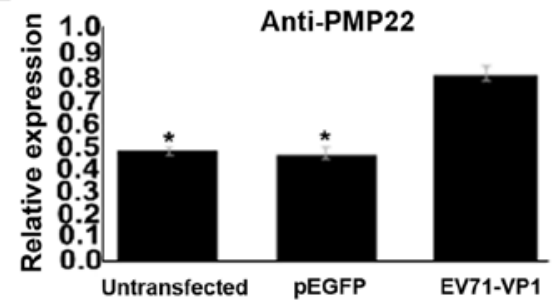

B

DAPI

GFP

PMP22

Merge

Untransfected
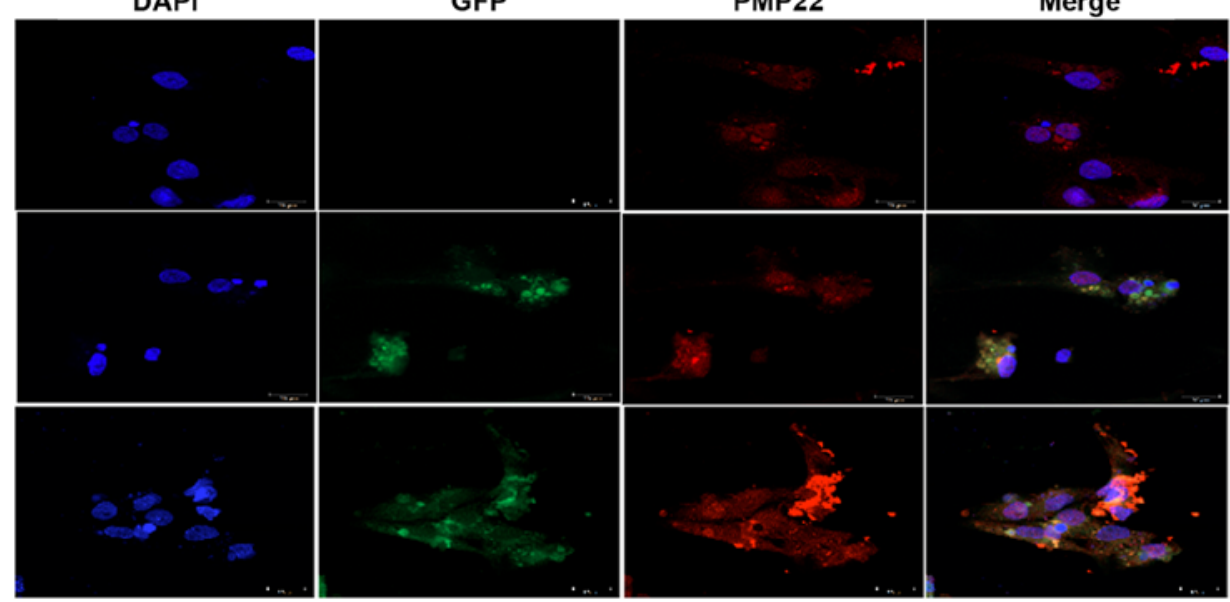

Figure 3. Effect of VP1 on PMP22 expression in mouse Schwann cells. Mouse Schwann cells were transfected with pEGFP-C3-VP1 for 48 h. Untransfected and empty vector pEGFP-C3-trasfected cells (pEGF) were used as blank and negative controls, respectively. (A) mRNA expression levels of PMP22. (B) Immunofluorescence analysis for PMP22. GFP expression was used to monitor the transfection efficacy. Magnification, x400. (C) Representative images and (D) quantification of relative PMP22 protein expression levels by western blotting. Data are expressed as means \pm standard error from three independent repeats. ${ }^{*} \mathrm{P}<0.05$ and ${ }^{* *} \mathrm{P}<0.01$ vs. EV71-VP1 group. VP1, structural viral protein 1; PMP22, peripheral myelin protein 22; GFP, green fluorescent protein; EV71, enterovirus 71 .
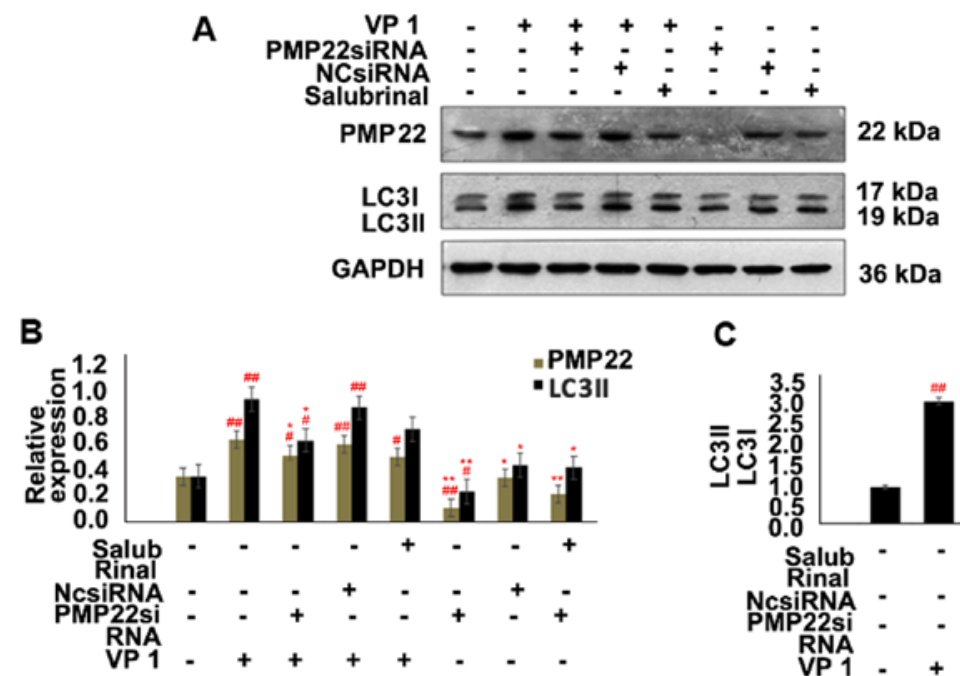

C

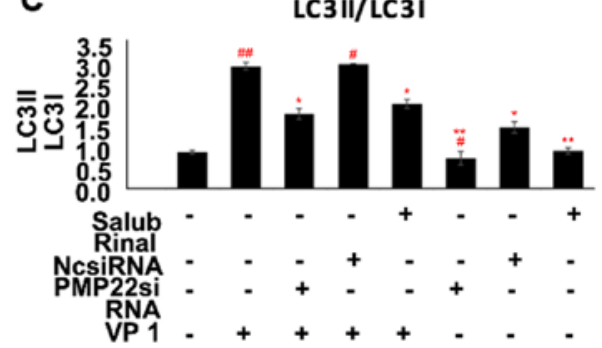

Figure 4. Effect of PMP22 knockdown on autophagy marker LC3II in mouse Schwann cells. Mouse Schwann cells were transfected with siPMP22. Untransfected and scramble siRNA (NCsiRNA)-transfected cells were used as blank and negative controls, respectively. Protein expression levels of PMP22 and LC3II were detected by western blot analysis. (A) Representative blots. (B) Quantification of PMP22 and LC3II protein expression. (C) Ratio of LC3II to LC3I. Data are expressed as mean \pm standard error from three independent repeats. ${ }^{*} \mathrm{P}<0.05$ and ${ }^{\# \#} \mathrm{P}<0.01$ vs. untransfected group; ${ }^{*} \mathrm{P}<0.05$ and ${ }^{* *} \mathrm{P}<0.01$ vs. VP1 group. PMP22, peripheral myelin protein 22; LC3I, microtubule-associated protein 1 light chain $3 \alpha$; LC3II, phosphatidylethanolamine-conjugated LC3I; si, small interfering; NC, negative control; VP1, structural viral protein 1.

since both PMP22 and ER stress are essential for activation of autophagy, the present study next sought to examine the relationship between PMP22 and ER stress in mouse Schwann cells using the selective ER stress inhibitor SAL. As presented in Fig. 6A, compared with the control groups, mRNA expression of PMP22 was significantly downregulated following SAL treatment. Consistently, markedly weak fluorescent staining of PMP22 was observed in SAL-treated mouse 

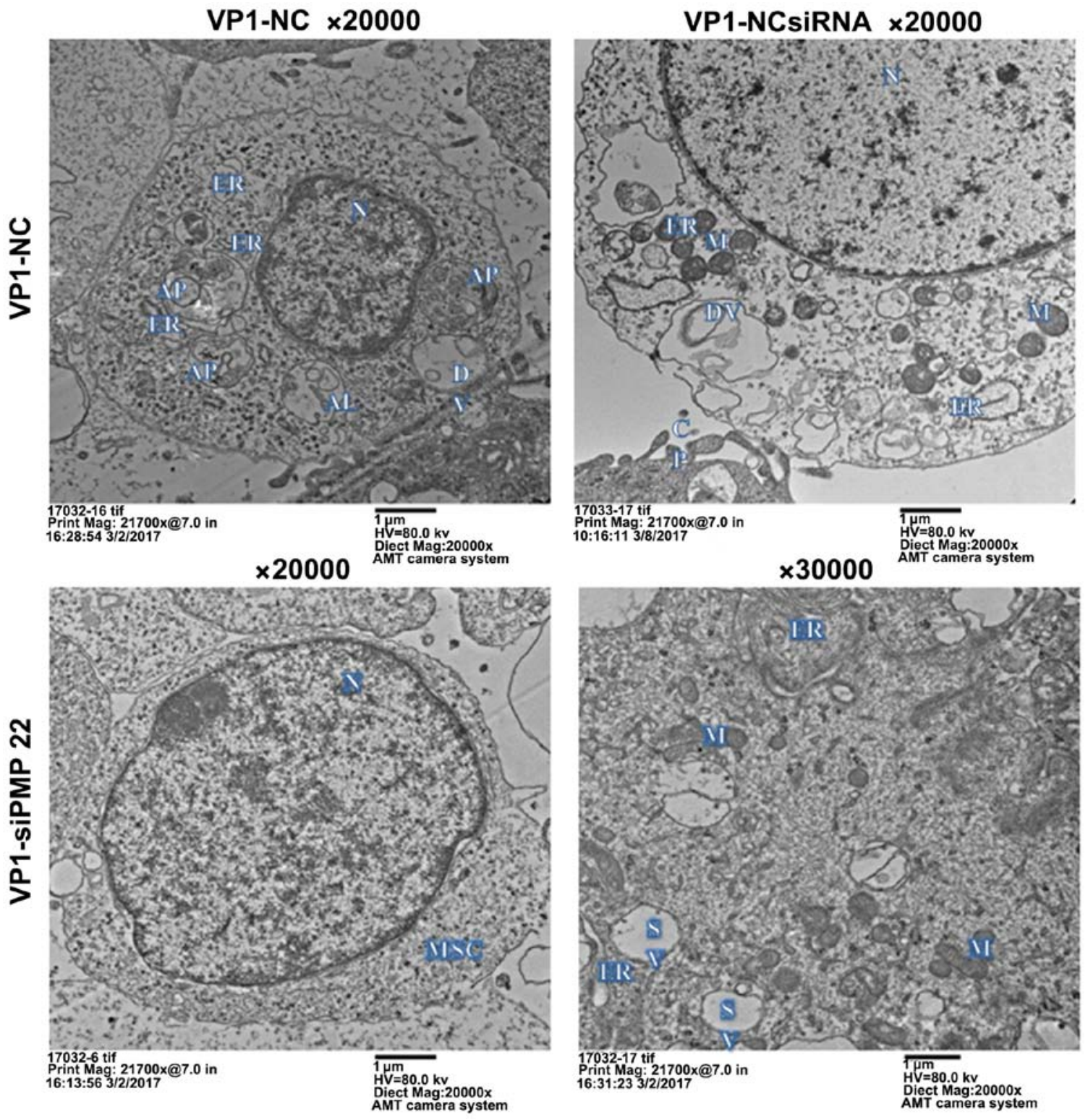

Figure 5. Effect of PMP22 knockdown on cellular and subcellular morphology. Mouse Schwann cells were transfected with siPMP22 for 48 h. Untransfected and scramble siRNA (NCsiRNA)-transfected cells were used as blank and negative controls, respectively. Representative transmission electron microscopic images depict subcellular structures of mouse Schwann cells. PMP22, peripheral myelin protein 22; si, small interfering; N, nucleus; M, mitochondrion; AP, autophagosome; AL, autolysosome; DV, degradation vesicles.

Schwann cells (Fig. 6B), suggesting that PMP22 expression in mouse Schwann cells is induced by ER stress. These results indicate that the VP1/ER stress/PMP22 signaling axis was an important component in mouse Schwann cells autophagy.

\section{Discussion}

The present study investigated the role and mechanism of the EV71 capsid protein VP1 in mouse Schwann cell autophagy, and demonstrated for the first time that VP1 promoted mouse Schwann cell autophagy through ER stress-mediated PMP22 upregulation, suggesting that the VP1/ER stress/PMP22 axis may serve as a novel potential target against EV71-induced neuronal disorder in severe HFMD cases.

The current results demonstrated that VP1 promoted mouse Schwann cell autophagy (Fig. 2), which is consistent with our previous findings (27). Nevertheless, the effect of VP1-induced autophagy on mouse Schwann cell survival remains unclear, because autophagy has dual roles in the nervous system. Excessive autophagy may be protective in chronic neurodegenerative diseases but detrimental in acute neural damages $(20,42)$. It has been reported that the inhibition of EV71-induced autophagy in human rhabdomyosarcoma cells inhibits cell apoptosis at the autophagosome formation and execution stages, but promotes apoptosis at the autophagosome-lysosome fusion stage. Furthermore, the inhibition of autophagy in the autophagosome formation stage decreases the release of EV71 viral particles, which is an effective strategy against virus infection (43). On the other hand, EV71-induced autophagy promotes viral replication in human rhabdomyosarcoma and neuroblastoma cells, and aggravates physiopathological parameters, including weight loss, disease symptoms, and mortality in mouse models $(25,26)$. Further in vitro and in vivo studies are required to clarify the exact role of VP1-induced autophagy in neuron cells. 
A

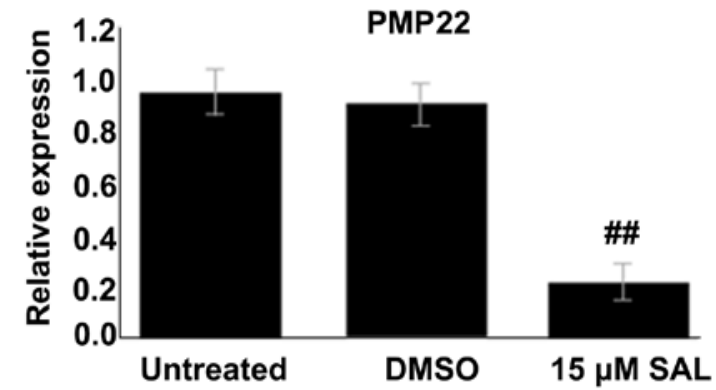

B
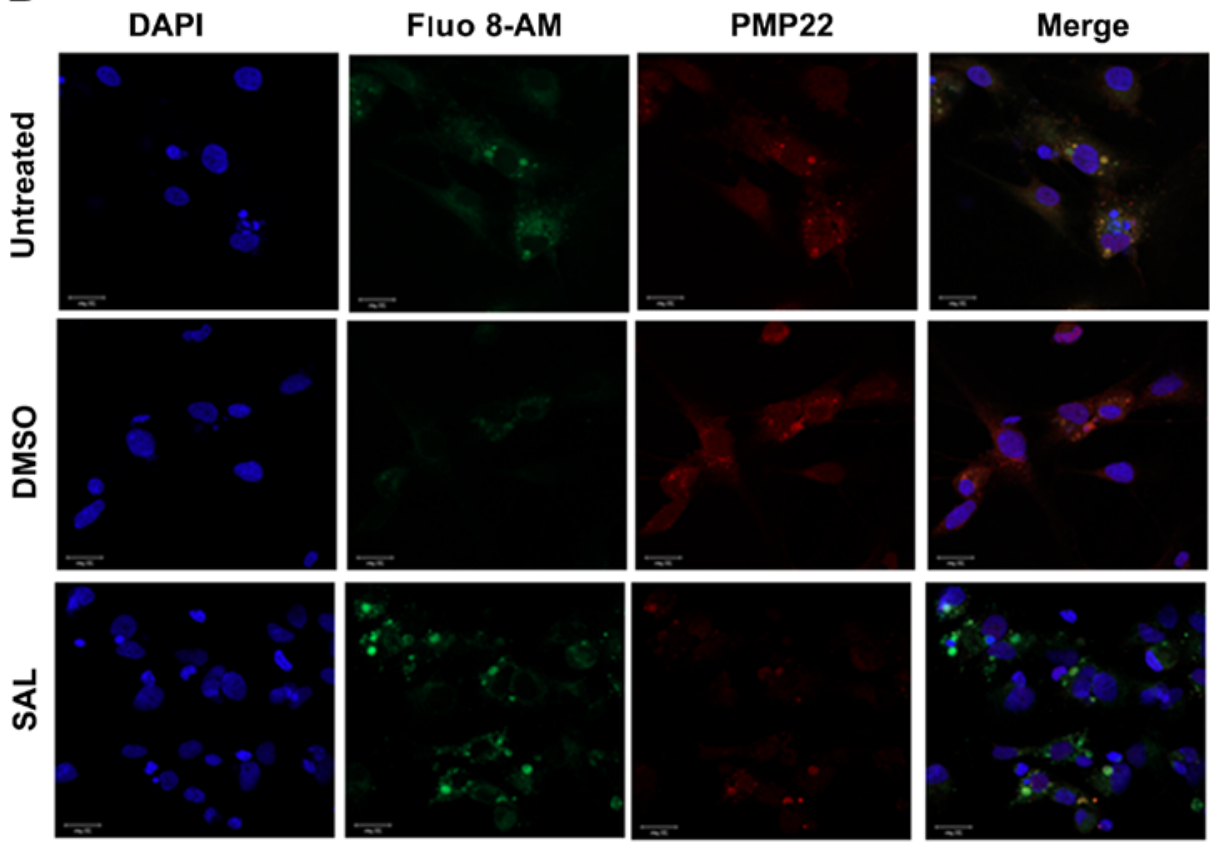

Figure 6. Effect of ER stress activation on PMP22 expression. Mouse Schwann cells were treated with $15 \mu$ M of the selective ER stress inhibitor SAL for $48 \mathrm{~h}$. Untreated and DMSO-treated cells were used as blank and negative controls. (A) mRNA expression levels of PMP22 were detected by reverse transcription-quantitative PCR. Data are expressed as mean \pm standard error of the mean from three independent repeats. ${ }^{\# \#} \mathrm{P}<0.01 \mathrm{vs}$. DMSO group. (B) Immunofluorescence analysis for PMP22 (red staining) in DMSO or SAL-treated mouse Schwann cells. Fluo-8 AM (green staining) was used as a positive control for the SAL-induced ER stress inhibition. Magnification, x400. ER, endoplasmic reticulum; PMP22, peripheral myelin protein 22; SAL, salubrinal.

The present results also demonstrated that VP1 overexpression upregulated the ER stress-associated protein PMP22 in mouse Schwann cells, suggesting an involvement of ER stress activation in VP1-induced autophagy. It is well-established that the presence of excessive or premature PMP22 in the ER induces ER stress $(37,38)$. Nevertheless, the effect of ER stress activation on PMP22 expression has not been investigated to date. The current data revealed for the first time that inhibition of ER stress significantly downregulated the expression of PMP22 in mouse Schwann cells (Fig. 6), suggesting that PMP22 is a downstream effector of ER stress. It seems that there is a positive feedback loop between ER stress and PMP22 in mouse Schwann cells. Furthermore, the present results demonstrated that in PMP22-deficient mouse Schwann cells there was no morphological signs of autophagy and the autophagy marker LC3II was downregulated (Figs. 4 and 5), suggesting that PMP22 was essential for mouse Schwann cell autophagy. Of note, in an EV71-infected mouse model, VP1 was found to co-localize with LC3 and/or autophagosome-like vesicles in neurons, and VP1 expression was positively correlated with LC3II expression, aggregation and autophagosome formation (26). Upregulation of LC3II expression was also observed in VP1-transfected 293 cells (27). Considering the regulatory role of VP1 in both mouse Schwann cell autophagy and PMP22 expression (Fig. 3), it can be concluded that the VP1/ER stress/PMP22 axis may have an important role in the activation of autophagy in mouse Schwann cells. Nevertheless, whether ER stress may contribute to autophagy independently of PMP22 still requires additional studies. In addition, EV71 could increase PMP22 expression through ER-dependent and independent mechanisms, which also requires additional studies. Finally, the present study was performed in mouse Schwann cells, while the mechanistic studies of EV71 have been mainly performed in tumor cells such as rhabdomyosarcoma. Caution is advised when comparing the results of the present study with the literature.

In summary, the present study demonstrated that mouse Schwann cell autophagy was activated by the EV71 capsid protein VP1. Mechanistically, the expression of the ER stress-associated protein PMP22 was significantly upregulated by VP1 overexpression, suggesting that ER stress-mediated PMP22 upregulation was at least partly responsible for VP1-induced autophagy activation. The VP1/ER stress/PMP22 axis may eventually serve as a target 
for novel therapeutic strategies against the neuronal damage induced by EV71 infection. Additional studies are necessary prior to clinical applications.

\section{Acknowledgements}

Not applicable.

\section{Funding}

No funding was received.

\section{Availability of data and material}

The data analyzed during the present study are available from the corresponding author on reasonable request.

\section{Authors' contributions}

SY and PL conceived and supervised the study. PL and DH designed experiments. HZ, JL and DW performed experiments. GL and JL provided new tools and reagents. SN developed new software and performed simulation studies. HY analyzed data. PL wrote the manuscript. SY and PL made manuscript revisions. All authors reviewed the results and approved the final version of the manuscript.

\section{Ethics approval and consent to participate}

The study was approved by the Ethics Committee of the Center for Disease Control and Prevention of Guangdong Province. All procedures performed involving human participants were in accordance with the ethical standards of the institutional and/or National Research Committee and with the 1964 Helsinki declaration and its later amendments or comparable ethical standards. Written informed consent was obtained from the family of the patient from whom EV71 was isolated for use of the clinical samples in research.

\section{Patient consent for publication}

Not applicable.

\section{Competing interests}

The authors declare that they have no competing interests.

\section{References}

1. Liu MY, Liu J, Lai W, Luo J, Liu Y, Vu GP, Yang Z, Trang P, $\mathrm{Li} \mathrm{H}$ and $\mathrm{Wu}$ J: Characterization of enterovirus 71 infection and associated outbreak of Hand, Foot, and Mouth disease in shawo of China in 2012. Sci Rep 6: 38451, 2016.

2. Wang Y, Zou G, Xia A, Wang X, Cai J, Gao Q, Yuan S, He G, Zhang S, Zeng M and Altmeyer R: Enterovirus 71 infection in children with hand, foot, and mouth disease in Shanghai, China: Epidemiology, clinical feature and diagnosis. Virol J 12: 83, 2015

3. Aswathyraj S, Arunkumar G, Alidjinou EK and Hober D: Hand, foot and mouth disease (HFMD): Emerging epidemiology and the need for a vaccine strategy. Med Microbiol Immunol 205: 397-407, 2016.

4. Shimizu H and Nakashima K: Surveillance of hand, foot, and mouth disease for a vaccine. Lancet Infect Dis 14: 262-263, 2014.
5. Zhang Y, Tan XJ, Wang HY, Yan DM, Zhu SL, Wang DY, Ji F, Wang XJ, Gao YJ, Chen L, et al: An outbreak of hand, foot, and mouth disease associated with subgenotype $\mathrm{C} 4$ of human enterovirus 71 in Shandong, China. J Clin Virol 44: 262-267, 2009.

6. Chong CY, Chan KP, Shah VA, Ng WY, Lau G, Teo TE, Lai SH and Ling AE: Hand, foot and mouth disease in Singapore: A comparison of fatal and non-fatal cases. Acta Paediatr 92: 1163-1169, 2003.

7. Sabanathan S, Tan le V, Thwaites L, Wills B, Qui PT and Rogier van Doorn H: Enterovirus 71 related severe hand, foot and mouth disease outbreaks in South-East Asia: Current situation and ongoing challenges. J Epidemiol Community Health 68: 500-502, 2014.

8. Solomon T, Lewthwaite P, Perera D, Cardosa MJ, McMinn P and Ooi MH: Virology, epidemiology, pathogenesis, and control of enterovirus 71. Lancet Infect Dis 10: 778-790, 2010.

9. Gui J, Liu Z, Zhang T, Hua Q, Jiang Z, Chen B, Gu H, Lv H and Dong C: Epidemiological characteristics and spatial-temporal clusters of hand, foot, and mouth disease in zhejiang province, China, 2008-2012. PLoS One 10: e0139109, 2015.

10. Xing W, Liao Q, Viboud C, Zhang J, Sun J, Wu JT, Chang Z, Liu F, Fang VJ, Zheng Y, et al: Hand, foot, and mouth disease in China, 2008-12: An epidemiological study. Lancet Infect Dis 14: 308-318, 2014.

11. Hsueh C, Jung SM, Shih SR, Kuo TT, Shieh WJ, Zaki S, Lin TY, Chang LY, Ning HC and Yen DC: Acute encephalomyelitis during an outbreak of enterovirus type 71 infection in Taiwan: Report of an autopsy case with pathologic, immunofluorescence, and molecular studies. Mod Pathol 13: 1200-1205, 2000.

12. Kuo RL, Kung SH, Hsu YY and Liu WT: Infection with enterovirus 71 or expression of its $2 \mathrm{~A}$ protease induces apoptotic cell death. J Gen Virol 83: 1367-1376, 2002.

13. Li ML, Hsu TA, Chen TC, Chang SC, Lee JC, Chen CC, Stollar V and Shih SR: The 3C protease activity of enterovirus 71 induces human neural cell apoptosis. Virology 293: 386-395, 2002.

14. Yang SD, Li PQ, Li YM, Li W, Lai WY, Zhu CP, Tao JP, Deng L, Liu HS, Ma WC, et al: Clinical manifestations of severe enterovirus 71 infection and early assessment in a Southern China population. BMC Infect Dis 17: 153, 2017.

15. Klionsky DJ: Autophagy: From phenomenology to molecular understanding in less than a decade. Nat Rev Mol Cell Biol 8: 931-937, 2007.

16. Mizushima N and Klionsky DJ: Protein turnover via autophagy: Implications for metabolism. Annu Rev Nutr 27: 19-40, 2007.

17. Ebrahimi-Fakhari D, Wahlster L, Hoffmann GF and Kolker S: Emerging role of autophagy in pediatric neurodegenerative and neurometabolic diseases. Pediatr Res 75: 217-226, 2014.

18. Ginet V, Spiehlmann A, Rummel C, Rudinskiy N, Grishchuk Y, Luthi-Carter R, Clarke PG, Truttmann AC and Puyal J: Involvement of autophagy in hypoxic-excitotoxic neuronal death. Autophagy 10: 846-860, 2014.

19. Lee JH, Yu WH, Kumar A, Lee S, Mohan PS, Peterhoff CM, Wolfe DM, Martinez-Vicente M, Massey AC, Sovak G, et al: Lysosomal proteolysis and autophagy require presenilin 1 and are disrupted by Alzheimer-related PS1 mutations. Cell 141: 1146-1158, 2010.

20. Klionsky DJ, Abdelmohsen K, Abe A, Abedin MJ, Abeliovich H, Acevedo Arozena A, Adachi H, Adams CM, Adams PD, Adeli K, et al: Guidelines for the use and interpretation of assays for monitoring autophagy (3rd edition). Autophagy 12: 1-222, 2016.

21. Tanida I, Ueno T and Kominami E: LC3 and autophagy. Methods Mol Biol 445: 77-88, 2008.

22. Benito-Cuesta I, Diez H, Ordonez L and Wandosell F: Assessment of autophagy in neurons and brain tissue. Cells 6: E25, 2017.

23. Huang SC, Chang CL, Wang PS, Tsai Y and Liu HS: Enterovirus 71-induced autophagy detected in vitro and in vivo promotes viral replication. J Med Virol 81: 1241-1252, 2009.

24. Lee YR, Wang PS, Wang JR and Liu HS: Enterovirus 71-induced autophagy increases viral replication and pathogenesis in a suckling mouse model. J Biomed Sci 21: 80, 2014.

25. Hu DD, Mai JN, He LY, Li PQ, Chen WX, Yan JJ, Zhu WD, Deng L, Wei D, Liu DH, et al: Glucocorticoids prevent enterovirus 71 capsid protein VP1 induced calreticulin surface exposure by alleviating neuronal ER stress. Neurotox Res 31: 204-217, 2017.

26. Rashid HO, Yadav RK, Kim HR and Chae HJ: ER stress: Autophagy induction, inhibition and selection. Autophagy 11: 1956-1977, 2015

27. Lin W and Popko B: Endoplasmic reticulum stress in disorders of myelinating cells. Nat Neurosci 12: 379-385, 2009. 
28. Lee WS, Yoo WH and Chae HJ: ER stress and autophagy. Curr Mol Med 15: 735-745, 2015.

29. Cai Y, Arikkath J, Yang L, Guo ML, Periyasamy P and Buch S: Interplay of endoplasmic reticulum stress and autophagy in neurodegenerative disorders. Autophagy 12: 225-244, 2016.

30. Zou X, Xu J, Yao S, Li J, Yang Y and Yang L: Endoplasmic reticulum stress-mediated autophagy protects against lipopolysaccharide-induced apoptosis in HL-1 cardiomyocytes. Exp Physiol 99: 1348-1358, 2014.

31. Cheng X, Liu H, Jiang CC, Fang L, Chen C, Zhang XD and Jiang ZW: Connecting endoplasmic reticulum stress to autophagy through IRE1/JNK/beclin-1 in breast cancer cells. Int J Mol Med 34: 772-781, 2014

32. Snipes GJ, Suter U, Welcher AA and Shooter EM: Characterization of a novel peripheral nervous system myelin protein (PMP-22/SR13). J Cell Biol 117: 225-238, 1992.

33. Pareek S, Suter U, Snipes GJ, Welcher AA, Shooter EM and Murphy RA: Detection and processing of peripheral myelin protein PMP22 in cultured Schwann cells. J Biol Chem 268: 10372-10379, 1993.

34. Ryan MC, Notterpek L, Tobler AR, Liu N and Shooter EM: Role of the peripheral myelin protein $22 \mathrm{~N}$-linked glycan in oligomer stability. J Neurochem 75: 1465-1474, 2000.

35. Dickson KM, Bergeron JJ, Shames I, Colby J, Nguyen DT, Chevet E, Thomas DY and Snipes GJ: Association of calnexin with mutant peripheral myelin protein-22 ex vivo: A basis for 'gain-of-function' ER diseases. Proc Natl Acad Sci USA 99: 9852-9857, 2002.
36. Hara T, Hashimoto Y, Akuzawa T, Hirai R, Kobayashi H and Sato K: Rerl and calnexin regulate endoplasmic reticulum retention of a peripheral myelin protein 22 mutant that causes type $1 \mathrm{~A}$ Charcot-Marie-Tooth disease. Sci Rep 4: 6992, 2014.

37. Shibutani ST and Yoshimori T: A current perspective of autophagosome biogenesis. Cell Res 24: 58-68, 2014.

38. Barth S, Glick D and Macleod KF: Autophagy: Assays and artifacts. J Pathol 221: 117-124, 2010.

39. Livak KJ and Schmittgen TD: Analysis of relative gene expression data using real-time quantitative PCR and the 2(-Delta Delta C(T)) method. Methods 25: 402-408, 2001.

40. Shi M, Zhou Y, Cao L, Ding C, Ji Y, Jiang Q, Liu X, Li X, Hou X, Peng $\mathrm{H}$ and Shi W: Expression of enterovirus 71 capsid protein VP1 in Escherichia coli and its clinical application. Braz J Microbiol 44: 1215-1222, 2013.

41. Lal SK, Kumar P, Yeo WM, Kar-Roy A and Chow VT: The VP1 protein of human enterovirus 71 self-associates via an interaction domain spanning amino acids 66-297. J Med Virol 78: 582-590, 2006.

42. Puyal J, Ginet V, Grishchuk Y, Truttmann AC and Clarke PG: Neuronal autophagy as a mediator of life and death: Contrasting roles in chronic neurodegenerative and acute neural disorders. Neuroscientist 18: 224-236, 2012.

43. Xi X, Zhang X, Wang B, Wang T, Wang J, Huang H, Wang J, Jin Q and Zhao Z: The Interplays between autophagy and apoptosis induced by enterovirus 71. PLoS One 8: e56966, 2013. 